\title{
Misaligned visions for improving graduate diversity: Student characteristics vs. systemic/cultural factors
}

\author{
Lindsay Owens, ${ }^{1}$ Benjamin M. Zwickl, ${ }^{2}$ Scott V. Franklin, ${ }^{2}$ and Casey W. Miller ${ }^{1}$ \\ ${ }^{1}$ School of Chemistry and Material Science, Rochester Institute of Technology, 84 Lomb Memorial Drive, Rochester, NY 14623 \\ ${ }^{2}$ School of Physics and Astronomy, Rochester Institute of Technology, 84 Lomb Memorial Drive, Rochester, NY 14623
}

(Dated: October 12, 2018)

\begin{abstract}
Physics departments are increasingly working to improve diversity in graduate programs by using more holistic strategies in their admission and retention practices. However, completion rates for traditionally underserved groups are still problematic. By understanding and accounting for faculty and student divergence on challenges faced in retention, graduate programs will be better equipped to enact change. In this study of two graduate programs (one physics and the other astrophysics), faculty and graduate students were asked why graduate students leave their program, and to identify ways to reduce attrition. While the goal of improving retention was shared between faculty and graduate students, their visions for how retention could be improved were not aligned. Faculty believed that retention could be improved by reforming admissions practices to select for students with attributes critical for success in graduate school, such as resilience. In contrast, the graduate students noted more systemic or socio-cultural factors as impacting retention.
\end{abstract}

\section{INTRODUCTION}

Underrepresented minority students, such as African American, LatinX, and Native American, are disproportionally low in graduate STEM programs, and accounted for $12 \%$ of STEM doctorates awarded annually [1]; moreover, retention rates for these underserved groups in graduate physical science and mathematics programs are currently around 50\% nationally [2], suggesting an urgent need for programs to address contributing factors. The Council of Graduate Schools reported that underrepresented groups are even more at risk for not completing their doctoral programs [2]. Some reasons for why graduate students withdraw from their programs have been identified and include financial hardship and a lack of a sense of community [2,3]. Conversely, some argued that student characteristics, such as under-preparation, are the primary cause for attrition [4]. However, prior research aggregated multiple disciplines and did not specifically address physics or astrophysics graduate programs. Understanding factors contributing to attrition within physics graduate programs is essential in order to suggest constructive changes to reduce attrition rates, especially for traditionally underrepresented groups.

The first step in institutional change according to Intentional Change Theory [5] is to recognize a problem, a step called "Imagining the Ideal Self". Only then can an organization begin the process of enacting positive change to address the issue. Within the first step, there are two parts, (1) acknowledging the problem, wherein there must be agreement across all stakeholders (i.e., faculty and graduate students) and (2) an image of the desired future (i.e., increased retention of all graduate students). If faculty and graduate students' views about the problems at hand differ then generating effective and lasting change to department practices is not possible, even if their image of a desired future is shared. Discussions about factors affecting retention need to occur at the departmental level, since different graduate programs may have their own unique set of strengths and challenges.

In this paper, two graduate programs (one physics and the other astrophysics) will be discussed. Both programs had recognized the need to address retention within their graduate programs and faculty shared a secondary goal of wanting to increase diversity in their graduate programs. The faculty and graduate students at each institution shared an aligned vision on the broader issue (poor retention) and on their ideal future (increased retention). This preliminary study will discuss the goals of this initial work as well as the larger project. Analysis of interviews from both students and faculty within each program will be presented showing that having a shared vision does not guarantee agreement on how to move forward towards their ideal future. Implications of misaligned visions among stakeholders will also be discussed.

\section{METHODS}

During an in-person visit to each institution, faculty and students were interviewed using separate though complementary protocols aimed at gathering an understanding of admission and retention practices. The first program (Program A), was an astrophysics graduate program in a private not-forprofit university in the northeast; five faculty participated (4 male, 1 female) in the interviews and six graduate students participated in a single focus group. The second program (Program B), was in a public university in the southeastern United States; six faculty (4 male, 2 female) participated in interviews and 11 graduate students participated in focus groups with 3-5 students per group.

The interview protocol for faculty covered a range of topics on admissions and retention practices to determine faculty readiness to incorporate holistic practices into their program. The protocol invited faculty to share their opinions on retention practices such as faculty and peer mentoring and creating a community of support. Also, faculty were asked to share 
their ideas about why students had left their graduate programs. Then, as a follow-up question, faculty were asked if any steps had been taken (by themselves or the department) to address known issues. The goal of these questions was twofold: first, to understand what faculty believed were factors contributing to attrition as well as possible solutions, and second, to gauge whether faculty perceived retention to be problematic enough to warrant change. Complementary to that, graduate students were asked to share why their peers had left the program or to share reasons why they had ever considered leaving the program. The students were also asked if any steps had been taken by the faculty or the department to address their concerns.

Each section of text was double-coded to identify: (1) if it was from the perspective of a faculty member or a graduate student; (2) the topic (e.g., coursework, advisors, etc.). This double-coding process allowed for direct comparison of faculty and student perspectives on topics relating to retention. Within each topic, the text was further analyzed to determine if the topic was considered to be a routine part of graduate school or in need of change. A codebook was developed by the first author while analyzing roughly half of the interviews. Then, new two transcripts were coded by both the first author and an undergraduate researcher using the developed codebook. An initial comparison of codes showed $75 \%$ agreement, and upon discussion reached over $90 \%$ agreement.

\section{RESULTS}

In both Programs, faculty predominately listed coursework and qualifying exams as reasons for students leaving their respective programs. Graduate students, on the other hand, cited a variety of systemic and cultural factors as reasons why their peers withdrew (or considered withdrawing) from their programs. Some examples included lack of belonging within their department and a lack of work-life balance. This paper will focus on faculty and students' contrasting views, whereas faculty focus on student deficiencies, students focus on the systemic issues regarding coursework (Program A) and the qualifying exam (Program B).

\section{A. Program A - Coursework}

At Program A, the core graduate coursework is completed during the first two years. Though all students took the same courses in this astrophysics program, the students came from a variety of undergraduate majors, such as astronomy, physics, and computational mathematics.

\section{Faculty}

The faculty at Program A were asked why graduate students leave their program in general, and "failing to com- plete the coursework" was a common theme. Many of the faculty commented mentioned specific cases where students had left their program due to coursework, either from poor performance, or inability to balance the time commitment required for courses along with other obligations (e.g., teaching labs and grading as part of their teaching assistantships). This was especially true for students pursuing terminal masters degrees, according to one faculty member, who said:"[terminal masters students] are typically not the better students, so when they encounter the classes, they're not as successful." When asked what kind of students were able to complete their Ph.D. programs, one faculty member summed up the general consensus of the faculty:

"I think it does go to issues of, kind of, persistence, hard work in a sense. [...] The students who work harder on the homework tend to be the students who also work harder on research, not because the skills are exactly alike, but just because it's a matter of how much time, and energy, and how good they are at problem-solving for that."

To address the issue of losing students still engaged in coursework, faculty sought to improve their admissions practice by selecting for students who have a strong undergraduate preparation in physics and mathematics, along with demonstrated potential to be self-motivated and self-learning. Faculty insisted that students with such qualities would be able to not only balance the demands of coursework along with other obligations, but would likely possess the skills needed to understand new content presented in the courses. The faculty were also working on a curriculum redesign that would reduce the number of core courses in order to appear more attractive to prospective students but did not mention how this redesign was influenced by or affected their current graduate students. Both improving admissions and curriculum redesigns seemed to be focused on ensuring that new students would be more successful in the program, and did not incorporate strategies to address retention issues for current graduate students.

\section{Graduate Students}

The graduate students at Program A mentioned that coursework was a source of stress that led some students to withdraw. However, the students focused solely on the instruction of the courses:

"I think the problem that we're all trying to struggle with, collectively, is, you've admitted a bunch of students with a various background but your professors are teaching them as the students they want to have and not the students that are in their class."

One example mentioned in the focus group was a course taught by a professor whose research focus was in mathematics. This professor assumed (according to the students) that the students in their course had similar levels of mathematical preparation to each other. This misunderstanding of student backgrounds led to the omission of certain math- 
ematical content within the course which was essential for being able to solve problems. One student explained, "the expectations of what we should know and what was actually being taught were not matching." According to the students, at the end of the course, most of them received a failing grade, and brought the matter to the attention of the department. When discussing this issue with senior graduate students, those peers suggested that the problem was the students themselves which suggested a lack of peer support from other graduate students:

"It makes a very difficult dynamic between you and your peers because before all the final grades were in, we were the pariahs of the department like, 'Why are you guys complaining about your classes this badly? None of us had this problem."”

\section{B. Program B - Qualifying Exam}

At Program B, all of the core graduate coursework was completed during students' first year in the program, with their qualifying exam occurring after their second term of coursework with an option for a second try six months later. The content level of material appearing the qualifying exam was set at the undergraduate level. According to the faculty, this approach made the exam less challenging, and would allow more students to pass.

\section{Faculty}

Faculty were convinced that the qualifying exam was challenging enough to act as a bottleneck for students in pursuit of their doctoral degrees, with around three people from each cohort (a cohort is typically 15-20 students) being dismissed from the program as a result of failing the qualifying exam twice. Knowing that the goal of the program was to improve retention and that failing the qualifier twice would cause students to leave the program, one faculty member who served on the "Qualitative Exam Committee" (i.e., faculty who write the qualifying exam) was asked about the type of student who would be most likely to pass the exam. Their primary emphasis was on a strong background in physics content and reasoning ability:

"So students, from my point of view, have to demonstrate their maturity in science, so they're already ready to do research. So this is about general reasoning. Now, of course, they should know fundamentals of physics, of theoretical physics, of particle physics. They have to know it. And have some experience in reasoning and apply it to make some conclusions to come to solutions."

In order to address the issue of losing students at the qualifying exam, faculty sought to improve their admissions practice by selecting for students who not only had a strong undergraduate preparation in physics, but also their ability to be able to use their reasoning skills and problem-solving skills to be an independent learner.

\section{Graduate Students}

The graduate students at Program B also saw the qualifier as a roadblock in their academic careers; however, they were focused on a perceived unfairness that the exam presented. For example, approximately $25 \%$ of the students who participated in the focus groups had also completed their undergraduate work at Program B and felt significantly more prepared for the contents of the qualifier. These students completed the undergraduate courses with the professors who were writing the exam (at the undergraduate level). One of these students said, "We had the opportunity to take [courses] here, and we had a test essentially cooked in the way that we know how to do," while another student added "we literally did all of those problems the year before and for practice. The person who made the exam [...] was most likely was the person who taught us this." However, for those whose undergraduate coursework occurred at another institution, they described the experience as quite stressful.

\section{DISCUSSION}

While the goal of improving retention was shared between faculty and graduate students, their visions for how retention could be improved were not aligned. Further, the contrast between focusing on individual characteristics vs. systematic/cultural factors emerged as a critical issue. Faculty at both programs focused on improving their admissions process as a means to address retention, while graduate students were more focused on systemic change.

\section{A. Program A}

At Program A, coursework was seen by both faculty and students as problematic, but for varying reasons. Many of the faculty assumed that students' poor performance in coursework was attributable to ill preparation, lack of drive, or lack of time-management skills, while graduate students attributed the problem to mismatched expectations of faculty. Specifically, the students believed that faculty assumed all students had equivalent preparation in undergraduate coursework, despite coming from a variety of backgrounds.

In order to address the issue of performance in coursework, the faculty sought to change the admissions process to select for students who were persistent and showed good problemsolving skills. These qualities were repeatedly mentioned from faculty at Program A because they believed that students with those qualities would not only persist through the rigorous coursework but through the demands of research as well. 
Conversely, the graduate students believed that their performance in the coursework would improve if faculty had an accurate understanding of the variety of background of their students because faculty would not skip essential content assuming students had prior exposure.

\section{B. Program B}

The faculty who participated in the interviews at Program B claimed that students who had left likely failed the qualifying exam. However, the focus of improving retention was not centered on the qualifying exam but rather on the admissions process. Many of the faculty believed the qualifier was able to discern students ready for the demands of doctoral research and those who were not; therefore, efforts to improve retention should focus on improving the selection of admitted students. Given that the content of the qualifying exam focused on undergraduate physics problems, it was interesting that the faculty perceived the qualifier as a tool to determine students readiness for research. This disconnect was furthered when the faculty suggested that students admitted to the program should ideally have similar qualities to students who typically pass the qualifier and complete their degree. These qualities included having good reasoning skills, being curious, and being self-motivated. Yet, faculty did not indicate how possessing such qualities aided students in passing the qualifying exam. While both a restructuring of the qualifying exam and changing admissions practices would lead to improved retention, the faculty's proposed solution of admitting students ready for research appeared to be disconnected from the actual content of the qualifying exam.

Students at Program B saw the entire qualifying exam process as unfair due to the perceived advantage of students whose undergraduate work was also completed at Program B. The student desired to have the "Qualitative Exam Committee" create an exam that was equal in difficulty for everyone.

\section{Limitations and Future Work}

This preliminary study only observed one astrophysics and one physics graduate program, both of which had selfselected into this study. These two programs did not provide a comprehensive representation of graduate programs across the country. From Program A, only five of 15 faculty and six of 25 students were able to participate in the interviews. Six of 60 faculty and 11 out of 80 students participated from Program B. Given the limited number of participants additional opinions on problems and solutions within each program may have not been captured in the data.

As part of this project, another physics graduate program has already participated in the interviews ( 5 faculty; 6 graduate students), and the next phase of this project involves expanding to additional institutions to determine if misaligned visions between stakeholders are unique to these two programs or are a more universal phenomena.

\section{CONCLUSIONS}

In order for physics graduate programs to begin the process of enacting positive changes to their retention strategies in order to address the attrition of students, especially those in underrepresented groups who may be more affected, all of the stakeholders must be agreement on the problem at hand and the desired outcome. In this study, two institutions recognized an ongoing issue with retention among their graduate students and faculty shared an additional goal of increasing diversity within their graduate programs. In both programs, there was a hidden disagreement between stakeholders about issues affecting retention of graduate students and subsequently how to best address those issues. Faculty sought to improve their admissions practice in order to have a positive effect on retention rates in the long-term, while graduate students were focused on improving systemic elements within the department. While focusing on student qualities (e.g., resilience, self motivation) in admissions is in alignment with holistic practices known to increase diversity, without also addressing issues of retention is remains unlikely that long-term diversity goals will be met. In order to move forward in enacting positive change within these programs, faculty and students need to engage in open dialogue about: the underlying issues, the best strategies with which to address those issues, developing feedback and evaluative means to assess progress, and a shared image of their desired future.

\section{A. Acknowledgements}

Support for this was provided by the National Science Foundation- 1633275. Thank you to Jacob Mekker for assisting with the inter-rater reliability of the qualitative data.
[1] H. Okahana, C. Klein, J. Allum, et al. Innov High Educ (2018).

[2] R. Sowell, J. Allum, and H. Okahana, Doctoral initiative on minority attrition and completion, (Council of Graduate Schools, Washington DC, 2015).

[3] B. Lovitts, Leaving the ivory tower (Rowman \& Littlefield Publishers, Lanham Maryland, 2001)
[4] L. Cassuto, Ph.D. attrition: how much is too much? The Chronicle of Higher Education (2013).

[5] R. E. Boyatzis, An overview of intentional change from a complexity perspective, Journal of Management Development 25, 7 (2006). pp. 607-623. 